\title{
Platelet Reactivity and Coagulation Markers in Patients with COVID-19
}

\author{
Adriadne J. Bertolin • Talia F. Dalçóquio • Rocío Salsoso • Remo H. de M. Furtado • Roberto Kalil-Filho • \\ Ludhmila A. Hajjar • Rinaldo F. Siciliano • Esper G. Kallás • Luciano M. Baracioli • Felipe G. Lima • \\ Roberto R. Giraldez - Cyrillo Cavalheiro-Filho • Alexandra Vieira • Célia M. C. Strunz • Robert P. Giugliano • \\ Udaya S. Tantry · Paul A. Gurbel · José C. Nicolau (D)
}

Received: March 22, 2021 / Accepted: May 20, 2021 / Published online: June 4, 2021

(C) The Author(s), under exclusive licence to Springer Healthcare Ltd., part of Springer Nature 2021

\section{ABSTRACT}

Introdution: COVID-19 is associated with an increased risk of thrombotic events. However, the contribution of platelet reactivity $(\mathrm{PR})$ to

Supplementary Information The online version contains supplementary material available at https:// doi.org/10.1007/s12325-021-01803-w.

A. J. Bertolin · T. F. Dalçóquio - R. Salsoso ·

R. H. de M. Furtado · R. Kalil-Filho

L. A. Hajjar - R. F. Siciliano - L. M. Baracioli .

F. G. Lima - R. R. Giraldez - C. Cavalheiro-Filho ·

A. Vieira · C. M. C. Strunz · J. C. Nicolau ( $\square)$ Faculdade de Medicina, Instituto do Coracao (InCor), Hospital das Clinicas HCFMUSP,

Universidade de Sao Paulo, Sao Paulo, Sao Paulo, Brazil

e-mail: jose.nicolau@incor.usp.br

R. H. de M. Furtado

Hospital Israelita Albert Einstein, Sao Paulo, Sao Paulo, Brazil

R. F. Siciliano · E. G. Kallás

Department of Infectious and Parasitic Diseases, University of São Paulo, Sao Paulo, Sao Paulo, Brazil

R. P. Giugliano · U. S. Tantry

TIMI Study Group, Cardiovascular Division, Department of Medicine, Brigham and Women's Hospital and Harvard Medical School, Boston, MA, USA

P. A. Gurbel

Sinai Center for Thrombosis Research and Drug Development, Sinai Hospital of Baltimore,

Baltimore, MD, USA the aetiology of the increased thrombotic risk associated with COVID-19 remains unclear. Our aim was to evaluate PR in stable patients diagnosed with COVID-19 and hospitalized with respiratory symptoms (mainly dyspnoea and dry cough), in comparison with a control group comprised of non-hospitalized healthy controls. Methods: Observational, case control study that included patients with confirmed COVID19 (COVID-19 group, $n=60$ ) and healthy individuals matched by age and sex (control group, $n=60$ ). Multiplate electrode aggregometry (MEA) tests were used to assess PR with adenosine diphosphate (MEA-ADP, low PR defined as $<53$ AUC), arachidonic acid (MEAASPI, low PR $<86$ AUC) and thrombin receptoractivating peptide 6 (MEA-TRAP, low PR $<97$ AUC) in both groups.

Results: The rates of low PR with MEA-ADP were $27.5 \%$ in the COVID-19 group and $21.7 \%$ in the control group $(\mathrm{OR}=1.60, p=0.20)$; with MEA-ASPI, the rates were, respectively, $37.5 \%$ and $22.5 \%(\mathrm{OR}=3.67, p<0.001)$; and with MEA-TRAP, the incidences were $48.5 \%$ and $18.8 \%$, respectively $(\mathrm{OR}=9.58, p<0.001)$. Levels of D-dimer, fibrinogen, and plasminogen activator inhibitor 1 (PAI-1) were higher in the COVID-19 group in comparison with the control group (all $p<0.05$ ). Thromboelastometry was utilized in a subgroup of patients and showed a hypercoagulable state in the COVID19 group. 
Conclusion: Patients hospitalized with nonsevere COVID-19 had lower PR compared to healthy controls, despite having higher levels of D-dimer, fibrinogen, and PAI-1, and hypercoagulability by thromboelastometry.

Trial Registration: ClinicalTrials.gov identifier, NCT04447131.

Keywords: COVID-19; Multiplate electrode aggregometry; Platelet reactivity;

Thromboelastometry

\section{Key Summary Points}

Why carry out this study?

Patients with COVID-19 have an increased risk for thrombotic events.

Abnormal coagulation markers, including higher levels of D-dimer and fibrinogen, and a prolongation of the prothrombin time, have been demonstrated in these patients.

\section{What was learned from the study?}

COVID-19 patients had lower platelet reactivity compared to healthy controls.

Platelet reactivity had a predictive value for total time of hospitalization $\leq 7$ or $>7$ days.

Our findings suggest that platelet reactivity has no role in the pathophysiology of the hypercoagulable state observed in patients with COVID-19.

\section{DIGITAL FEATURES}

This article is published with digital features, including a summary slide, to facilitate understanding of the article. To view digital features for this article go to https://doi.org/10.6084/ m9.figshare.14575521.

\section{INTRODUCTION}

The coronavirus disease 2019 (COVID-19) pandemic has affected more than 144 million people worldwide as of April 23, 2021 [1]. Patients with COVID-19 are at increased risk of thrombotic events, and usually have abnormal coagulation markers, including high levels of Ddimer and fibrinogen, as well as prolonged prothrombin time [2-5].

On the other hand, evidence about the role of platelets in the pathophysiology of thrombosis among patients with COVID-19 is scant. Small studies have suggested different patterns of platelet reactivity (PR) [6, 7]. Additionally, the use of the IIb/IIIa inhibitor, tirofiban, was associated with lower mortality, [8] whereas thrombocytopenia was associated with increased mortality in prior observational studies $[9,10]$.

Therefore, the contribution of PR in the aetiology of the increased thrombotic risk associated with COVID-19 remains uncertain. The main objective of this study is to evaluate PR in stable patients diagnosed with COVID-19 and hospitalized with respiratory symptoms, in comparison with a control group comprised of healthy controls.

\section{METHODS}

\section{Study Design, Setting, and Population}

This is an observational, case-control study conducted at the Heart Institute (InCor/ HCFMUSP) and the Central Institute (CI/ HCFMUSP) of the University of Sao Paulo Medical School which enrolled patients with confirmed COVID-19 (case group, $n=60$ ), matched by age and sex with a group comprised of healthy non-hospitalized individuals (control group, $n=60$ ). The inclusion phase was between April and July, 2020.

The main inclusion criteria for the case group were hospitalization due to respiratory symptoms with a maximum of $72 \mathrm{~h}$ between hospital arrival and blood collection for laboratory assessments, age $>18$ years, and stable at hospitalization. SARS-CoV-2 infection was 
confirmed by positive quantitative RT-PCR $(n=52)$ or serology $(n=8)$, in accordance with the current standards of care. The main exclusion criteria included known platelet dysfunction and/or platelet count $<100,000 / \mathrm{mm}^{3}$ or $>450,000 / \mathrm{mm}^{3}$, terminal illness, known liver disease (such as cancer, chronic hepatitis, cholestatic liver disease, cirrhosis) or coagulation disorder, hematocrit $<34 \%$ or $>55 \%$, current use of antiplatelet therapy and/or anticoagulants (except acetylsalicylic acid and prophylactic heparin), and need for invasive mechanical ventilation or high flow oxygen. The control group included non-hospitalized healthy volunteers with no previous history of COVID-19 or any other known chronic disease (with the exception of hypertension, obesity, and dyslipidemia), and negative serology for SARS-CoV-2. According to institutional standards of care, all patients hospitalized with COVID-19 received prophylatic enoxaparin subcutaneously at doses of $40 \mathrm{mg}$ QS (if weight $<80 \mathrm{~kg}$ ), $60 \mathrm{mg}$ QD (if weight $80-120 \mathrm{~kg}$ ) and $40 \mathrm{mg}$ BID (if weight $>120 \mathrm{~kg}$ ). Aspirin was used (for primary or secondary prevention of coronary disease, according to the attending physicians) in 7 patients, all at the dose of $100 \mathrm{mg}$ QD by the oral route.

Decisions to discharge the patient or not were left at the discretion of the attending physician. However, health care professionals taking care of the patients were not aware of the results of $\mathrm{PR}$, nor were investigators from the study involved in medical assistance to these patients. Therefore, decisions to discharge the patient or not likely were not influenced by knowledge of the platelet function tests.

This study was approved by the Institutional Review Board (Comissão de Ética para Análise de Projetos de Pesquisa do HCFMUSP-CAAE: 34121120.2.0000.0068), and was conducted in accordance with the declaration of Helsinki of 1964 and its later amendments. All participants provided written informed consent. The study was registered at ClinicalTrials.gov (NCT04447131) and was designed to analyze three groups of patients hospitalized with respiratory symptoms at primary diagnosis: COVID-19, influenza, and neither COVID-19 nor influenza. Here, we report the results of the comparison between patients with COVID-19 versus healthy non-hospitalized individuals. The inclusions for the other groups are still ongoing.

\section{Study Procedures}

Individuals from both groups were initially submitted to a medical interview to assess the eligibility criteria. COVID-19 was confirmed by RT-PCR $(n=52)$ or sorology $(n=8)$. If eligible, individuals from the control group were tested for qualitative detection of IgG and IgM antibodies to SARS-Cov2 by immunochromatography (ECO Diagnosis, Corinto, Brazil).

Patients included in the case group had blood samples collected for the evaluation of platelet function and other coagulation parameters at baseline, as described in the sequence.

\section{Coagulation Markers}

Hemoglobin, platelet number, and mean platelet volume (MPV) were evaluated with an automated counter, ADVIA 120 (Siemens Healthcare Diagnostics, Newark, USA). Soluble P-selectin and plasminogen-activator-inhibitor (PAI-1) were evaluated using an enzyme-linked immunosorbent assay (IBL International, Hamburg, Germany). D-Dimer was evaluated by the STA $^{\mathrm{TM}}$ commercial kit, Liatest TM D-Di; fibrinogen was evaluated by the $\mathrm{STA}^{\mathrm{TM}}$, STA Compact $^{\mathrm{TM}}$, and STA Satellite ${ }^{\mathrm{TM}}$ kit; activated partial thromboplastin time (aPTT) and prothrombin time (PT) were determined using the TcoagCLOT kit with TcoagDestinyMax equipment); immature platelet fraction and count were determined by Sysmex XN-2000 ${ }^{\mathrm{TM}}$, which is based on the principle of hemocytometry.

\section{Platelet Reactivity}

For the analysis of PR in both groups, blood samples were taken after a 15 -min rest in a sitting position, with light tourniquets and short and moderate phlebostasis of the antecubital vein using 19-gauge butterfly needles. The first $3 \mathrm{~mL}$ were collected in hirudin S-Monovettes ${ }^{\circledR}$ tubes (Sarstedt, Nuembrecht, Germany). 
After 30 min of rest, MEA was performed on the Multiplate system, as recommended by the manufacturer, adding $0.3 \mathrm{~mL}$ of pre-heated $\left(37^{\circ} \mathrm{C}\right)$ isotonic saline to $0.3 \mathrm{~mL}$ of whole blood in the Multiplate test cell, followed by incubation for $3 \mathrm{~min}$ at $37^{\circ} \mathrm{C}$. Then, aggregation was induced by the addition of arachidonic acid (ASPItest), ADP (ADPtest), or TRAP-6 (TRAPtest). The aggregation response over $6 \mathrm{~min}$ was expressed by the area under the curve (AUC) [11]. The median time between blood sampling and the analyses was 115 min (IQR 60-145).

\section{Viscoelastic Properties}

Viscoelastic properties of the clot, such as clot formation (coagulation time and clot formation time), clot strength (maximum clot firmness), Alpha angle, and clot lysis were determined using the ROTEM delta device (Instrumentation Laboratory, Bedford, MA, USA) $[12,13]$.

\section{Study Endpoints}

The primary objective of the study was to compare PR analyzed by the MEA-ADPtest in hospitalized patients diagnosed with COVID-19 versus healthy non-hospitalized controls.

The main secondary endpoints were the comparison between COVID-19 patients versus healthy individuals regarding: (1) PR analyzed by the following: MEA-ASPI, MEA-TRAP tests; (2) immature platelet fraction and count; and (3) coagulation tests.

Other secondary objectives included: (1) correlation between ADP ASPI, TRAP-induced $\mathrm{PR}$, and composite clinical outcome of death from any cause, thrombotic events [defined as arterial or venous thrombosis, such as acute myocardial infarction (AMI), stroke, and pulmonary thromboembolism diagnosed according to the current guidelines] [14, 15], need for intensive care unit (ICU) admission, and need for intubation during hospitalization; and (2) correlation between ADP-, ASPI- and TRAP-induced PR and length of hospital stay $(\geq 7$ vs. $<7$ days, which was the median length of hospital stay in the COVID-19 group).

\section{Statistical Analysis}

The sample size for the comparison between COVID-19 patients and healthy individuals was calculated considering a mean PR of $81 \pm 16.7$ AUC in the control group according to a previous report with a similar population [16], and assuming a $20 \%$ higher PR in the COVID-19 group. Considering a power of $98 \%$, a two-tailed alpha of 0.01 , and an estimated loss of $30 \%$, the calculated sample size was 120 individuals (60 patients in each group).

Data are presented as absolute number and percentage for categorical variables, and means [ \pm standard deviations (SD), if Gaussian distribution] or medians [interquartile ranges (IQR), if non-Gaussian distribution], for continuous variables.

Categorical variables were compared between groups using the Chi-square test or Fisher's exact test when indicated.

Continuous variables were assessed for Gaussian or non-Gaussian distribution using the Kolmogorov-Smirnov or Shapiro-Wilk tests. To compare independent samples, Student's $t$ tests for independent samples (Gaussian distribution) or Mann-Whitney U tests (nonGaussian distribution) were used. For the correlation between platelet reactivity by MEAADP and the composite clinical outcome, logistic regression was utilized. To compare paired samples, Student's $t$ test for paired samples (if Gaussian distribution) or the Wilcoxon test (if non-Gaussian distribution) was used.

In order to compare platelet aggregation as continuous variables between both groups of interest, we have also developed multivariable linear regression models. The models were adjusted for the following independent variables: diabetes, presence of obesity $\left(\mathrm{BMI} \geq 30 \mathrm{~kg} / \mathrm{m}^{2}\right)$, and coronary artery disease (CAD). These variables were included in the model because they were significantly different between the two groups. For comparison of platelet aggregation as categorical variable (low or not PR), a logistic regression model was developed adjusting for the same covariates.

In assessing the correlation between PR and immature platelet index or length of hospital 
stay, the Spearman's correlation test was utilized.

Receiver operating characteristic (ROC) curves were estimated for MEA-ADP, MEA-ASPI, MEA-TRAP, and hospital length of stay $\geq 7$ or $<7$ days.

Due to the nature of this study, no adjustment for multiplicity was carried out, and there was no imputation for missing data since there was no missing observation for the primary endpoint. All tests are two-tailed and a $p$ value below 0.05 was considered as statistically significant. The statistical analyses were performed using SPSS 23.0 (Microsoft, Chicago, IL, USA) and StataTM v.15.1 (Statacorp, College Station, TX, USA).

\section{RESULTS}

A total of 685 hospitalized patients were screened between April and July 2020, among whom 120 patients were included in the study. The main reasons for exclusion were time of hospitalization $>72 \mathrm{~h} \quad(n=174)$, use of antiplatelet/anticoagulant other than low dose aspirin or prophylactic heparin $(n=130)$, and refusal to consent $(n=128)$ (Fig. 1$)$.

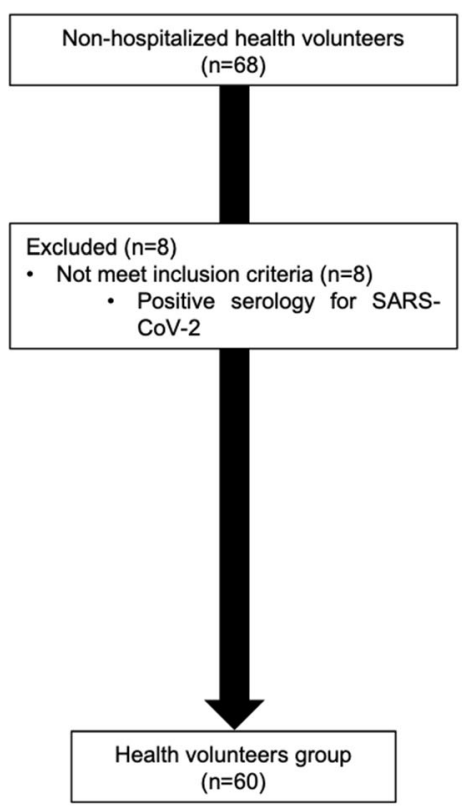

Fig. 1 Flowchart of selection criteria
The baseline characteristics of the population are depicted in Table 1. As expected, the COVID-19 and control groups were well matched with respect to age and sex. Higher body mass index, and higher prevalence of diabetes and coronary artery disease were observed in the COVID-19 group. As pointed out previously, all patients in the COVID-19 group were on prophylactic heparin and 7 were on lowdose aspirin.

The respiratory symptoms most frequently presented in the COVID-19 group were dyspnoea $(80 \%)$ and dry cough $(70 \%)$. The rates of the other symptoms are depicted at Suppl. Table 1.

\section{Platelet Reactivity}

As shown in Suppl. Table 2, the mean value of PR by MEA-ADP was $53.8 \pm 24.21$ AUC for the COVID-19 group and 54.4 \pm 20.45 AUC for the control group, $(p=0.88)$; by MEA-ASPI, the figures were $63.71 \pm 29.18$ AUC and $87.46 \pm 25.52$ AUC, respectively $(p<0.001)$, and by MEA-TRAP, $72.23 \pm 20.97$ AUC and $100 \pm 23.27$ AUC, respectively $(p<0.001)$. The results did not change substantially in a

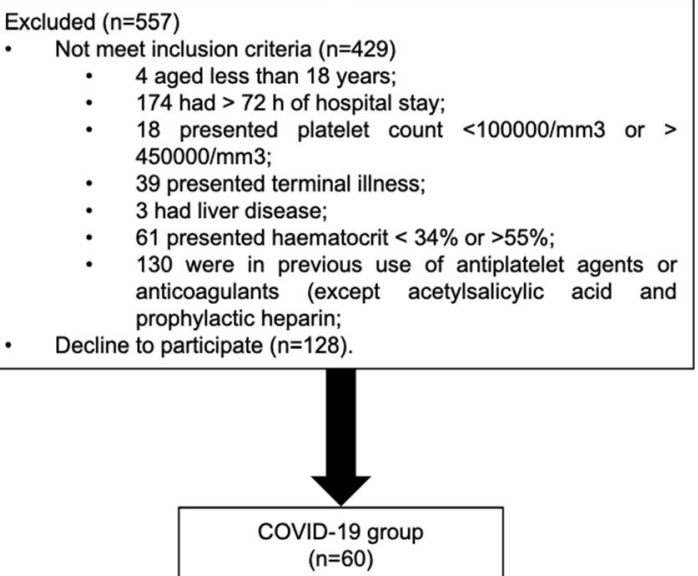

COVID-19 within $72 \mathrm{~h}$ hospitalization $(n=617)$

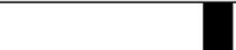


Table 1 Baseline characteristics of the global population

\begin{tabular}{|c|c|c|c|}
\hline & COVID-19 $(n=60)$ & Control $(n=60)$ & $p$ value \\
\hline Age (years), mean $( \pm S D)$ & $52.41( \pm 15.09)$ & $51.13( \pm 14.28)$ & $0.63^{\mathrm{a}}$ \\
\hline Male sex, $n(\%)$ & $31(51.6)$ & $31(51.67)$ & $0.92^{\mathrm{b}}$ \\
\hline On supplemental oxygen, $n(\%)$ & $44(73.3 \%)$ & NA & NA \\
\hline $\mathrm{FiO}_{2}$ among those on supplemental oxygen, median (IQR) & $0.28(0.25-0.34)$ & NA & NA \\
\hline Hypertension, $n(\%)$ & $28(46.6)$ & $18(30)$ & $0.60^{\mathrm{b}}$ \\
\hline Diabetes, $n(\%)$ & $18(30)$ & $1(1.67)$ & $<0.001^{\mathrm{b}}$ \\
\hline Dyslipidemia, $n(\%)$ & $10(16.67)$ & $5(8.33)$ & $0.16^{\mathrm{b}}$ \\
\hline BMI $\left(\mathrm{Kg} / \mathrm{m}^{2}\right)$, mean $( \pm \mathrm{SD})$ & $31.9( \pm 6.4)$ & $28.4( \pm 4.9)$ & $0.001^{\mathrm{a}}$ \\
\hline $\mathrm{BMI} \geq 30\left(\mathrm{Kg} / \mathrm{m}^{2}\right), n(\%)$ & $33(55)$ & $21(35)$ & $0.02^{\mathrm{b}}$ \\
\hline $\mathrm{CAD}, n(\%)$ & $5(8.3)$ & $0(0)$ & $0.02^{\mathrm{b}}$ \\
\hline $\mathrm{AF}, n(\%)$ & $0(0)$ & $0(0)$ & NA \\
\hline Valve disease, $n(\%)$ & $0(0)$ & $0(0)$ & NA \\
\hline Heart failure, $n(\%)$ & $0(0)$ & $0(0)$ & NA \\
\hline Heart transplantation, $n(\%)$ & $2(3.33)$ & $0(0)$ & $0.15^{\mathrm{b}}$ \\
\hline Stroke, $n$ (\%) & $0(0)$ & $0(0)$ & NA \\
\hline PAOD, $n(\%)$ & $0(0)$ & $0(0)$ & NA \\
\hline $\mathrm{CKD}, n(\%)$ & $1(1.67)$ & $0(0)$ & $1.0^{\mathrm{b}}$ \\
\hline
\end{tabular}

$B M I$ body mass index, $C A D$ coronary artery disease, $A F$ atrial fibrillation, $P A O D$ peripheral arterial occlusive disease, $C K D$ chronic kidney disease, $\mathrm{FiO}_{2}$ fraction of inspired oxygen (ranges from 0 to 1), IQR interquartile range, $S D$ standard deviation, $N A$ not available

a Student's $t$ test

${ }^{\mathrm{b}}$ Chi-square test

multivariable linear regression model (Suppl. Table 3).

As can be seen in Table 2, patients from the COVID-19 group had a higher prevalence of low PR (the cut-off points for low PR were defined as values under the normal range, 17-19), which were statistically significant for MEA-ASPI and MEA-TRAP, but not for MEA-ADP. The results did not change substantially in a multivariable logistic regression model (Suppl. Table 4). Eight patients had COVID-19 confirmed by serology (the disease was confirmed by positive quantitative RT-PCR in the remaining 52 patients); excluding those 8 patients did not materially change the main results (Suppl. Tables 5 and 6). Additionally, after excluding 7 patients taking aspirin, the results remained similar: the mean PR between the COVID-19 and control groups were $56.4 \pm 24.3$ AUC and $54.7 \pm 20.5$ AUC, $p=0.69 \quad$ (MEA-ADP); $67.4 \pm 28.1 \quad$ AUC and $88.1 \pm 25.3$ AUC, $p<0.001$ (MEA-ASPI); and $73.6 \pm 21.6$ AUC and $100.8 \pm 22.8$ AUC, $p<0.001$ (MEA-TRAP), respectively.

Suppl. Tables 7, 8, and 9 show the PR in the two groups excluding medications that could influence PR. The results were similar to those obtained for the whole population.

\section{Laboratory Analyses}

Table 3 shows the results for the other prespecified laboratory analyses. Patients with 
Table 2 Platelet reactivity in COVID-19 and control groups

\begin{tabular}{|c|c|c|c|c|c|c|}
\hline & $n$ & COVID-19 & $n$ & Control & OR $(95 \% \mathrm{CI})$ & $p$ value \\
\hline Low PR by MEA-ADP ${ }^{\mathrm{a}}, n(\%)$ & 60 & $33(55)$ & 60 & $26(43.33)$ & $1.60(0.80-3.30)$ & $0.20^{\mathrm{d}}$ \\
\hline Low PR by MEA-ASPI ${ }^{\mathrm{b}}, n(\%)$ & 60 & $45(75)$ & 60 & $27(45)$ & $3.67(1.69-7.96)$ & $<0.001^{\mathrm{d}}$ \\
\hline Low PR by MEA-TRAPc, $n(\%)$ & 56 & $49(81.67)$ & 45 & $19(31.67)$ & $9.58(3.57-27.74)$ & $<0.001^{\mathrm{d}}$ \\
\hline
\end{tabular}

$P R$ platelet reactivity, MEA multiplate electrode aggregometry, ADP adenosine diphosphate, ASPI aspirin, TRAP thrombin receptor-activating peptide $6, O R$ odds ratio, $C I$ confidence interval, $A U C$ area under the curve

a MEA-ADP < 53 AUC

b MEA-ASPI < 86 AUC

c MEA-TRAP < 97 AUC

d Chi-square

Table 3 Laboratory findings

\begin{tabular}{|c|c|c|c|c|c|}
\hline & $n$ & COVID-19 & $n$ & Control & $p$ value \\
\hline Hemoglobin $(\mathrm{g} / \mathrm{dL})$, mean $( \pm \mathrm{SD})$ & 60 & $13.32( \pm 1.63)$ & 60 & $14.35( \pm 1.56)$ & $<0.001^{\mathrm{a}}$ \\
\hline Leukocytes $\left(10^{3} / \mathrm{mm}^{3}\right)$, median (IQR) & 60 & $6895(5010-6895)$ & 60 & $6595(5530-7612)$ & $0.33^{\mathrm{b}}$ \\
\hline Lymphocytes $\left(10^{3} / \mathrm{mm}^{3}\right)$, median (IQR) & 60 & $1091(845-1424)$ & 60 & $2088(1604-2553)$ & $<0.001^{\mathrm{b}}$ \\
\hline Platelets $\left(10^{3} / \mathrm{mm}^{3}\right)$, median (IQR) & 60 & $236.5(170.1-314.5)$ & 60 & $242.5(206.8-292.3)$ & $0.75^{\mathrm{b}}$ \\
\hline MPV(fL), median (IQR) & 60 & $10.5(9.6-10.9)$ & 60 & $9.8(9.1-10.4)$ & $<0.001^{\mathrm{b}}$ \\
\hline Immature platelet fraction (\%), median (IPF) & 52 & $4.0(3.0-5.9)$ & 56 & $3.55(2.6-5.3)$ & $0.17^{\mathrm{b}}$ \\
\hline Immature platelet count $\left(103 / \mathrm{mm}^{3}\right)$, mean $( \pm S D)$ & 52 & $1098.6( \pm 569.1)$ & 56 & $921.2( \pm 488.1)$ & $0.08^{\mathrm{a}}$ \\
\hline P-selectin (ng/mL), median (IQR) & 55 & $79.9(62.1-106.7)$ & 57 & $80.7(64.7-108.0)$ & $0.53^{\mathrm{b}}$ \\
\hline PAI-1 (ng/mL), median (IQR) & 52 & $595.4(427.3-897.0)$ & 57 & $478.8(312.2-663.0)$ & $0.02^{\mathrm{b}}$ \\
\hline D-Dimer (ng/mL), median (IQR) & 57 & $966.0(624.0-1431.0)$ & 56 & $270.0(270.0-317.5)$ & $<0.001^{\mathrm{b}}$ \\
\hline Fibrinogen $(\mathrm{mg} / \mathrm{dL})$, mean $( \pm \mathrm{SD})$ & 49 & $615.4( \pm 152.1)$ & 58 & $326.3( \pm 74.1)$ & $<0.001^{\mathrm{b}}$ \\
\hline aPTT (s), median (IQR) & 58 & $35.2(32.1-40.0)$ & 60 & $34.8(32.8-38.5)$ & $0.72^{\mathrm{b}}$ \\
\hline PT (s), median (IQR) & 59 & $13.6(12.9-14.3)$ & 60 & $13.5(13.1-14.1)$ & $0.89^{\mathrm{b}}$ \\
\hline Interleukin-6, median (IQR) & 57 & $7.90(2.70-31.1)$ & 55 & $2.70(2.70-3.80)$ & $<0.001^{\mathrm{b}}$ \\
\hline
\end{tabular}

$M P V$ mean platelet volume, $P A I-1$ type 1 plasminogen activator inhibitor, aPTT activated partial thromboplastin time, $P T$ prothrombin time, $S D$ standard deviation, $I Q R$ interquartile range

a Student's $t$ test

b Mann-Whitney test

COVID-19 had lower hemoglobin and lymphocytes levels ( $p<0.001$ for both) compared to the control group; however, higher MPV $(p<0.001)$ PAI-1 $(p=0.02)$, D-dimer $(p<0.001)$, fibrinogen $(p<0.001)$, and interleukin-6 $(p<0.001)$ were observed in the COVID-19 group compared to the control group. There were no significant differences between the groups regarding the other analyzed variables. 


\section{Viscoelastic Properties}

As shown in Table 3, patients with COVID-19 had greater clotting times $(p=0.03)$, probably related to the use of heparin in this group. Additionally, in comparison with the control group, the COVID-19 group had lower median clot formation time $(p=0.04)$, higher alfa angle $(p=0.02)$, and higher maximum clot firmness $(p=0.03)$ (Table 4).

\section{Correlation Between Platelet Reactivity and Length of Hospitalization or In- Hospital Outcomes}

The results for the correlation between the MEA tests and the length of hospital stay $\leq 7$ versus $>7$ days are shown in Table 5 . Significant inverse correlations were observed for MEA-ADP and MEA-TRAP, but not for MEA-ASPI. Considering hospital length of stay as a continuous variable, a similar result was obtained, with significant correlations for MEA-ADP and MEATRAP, but not for MEA-ASPI (Suppl. Table 10). There were no correlations between immature platelets function and count with hospital length of stay (Table 5).

The correlation between PR and clinical outcomes in both groups is shown in Suppl. Table 11. PR was consistently lower in patients with events but reached statistical significance only with MEA-ADP for the composite endpoint of mortality, thrombotic events, and need for intensive care admission or mechanical ventilation. By multivariable logistic regression, patients with low platelet reactivity by MEAADP showed an OR $=4.0$ (95\% CI 0.99-16.25, $p=0.053$ ) for the incidence of the composite endpoint during hospitalization.

Prediction of length of hospital stay $>7$ days based on PR measured by MEA with different agonists is shown in Fig. 2. MEA-ADP and MEATRAP had good and significant predictive values for shorter hospitalization, with AUCs of 0.73 $(p=0.004)$ and $0.76(p=0.001)$, respectively. Conversely, the predictive value for MEA-ASPI was poor.

\section{DISCUSSION}

In this study, contrary to our expectations, a lower PR was observed in patients with COVID19 in comparison with healthy individuals. To the best of our knowledge, there are only two published studies addressing this question. The first analyzed only 10 healthy individuals and a mix of 15 COVID-19 stable patients and patients with severe disease hospitalized in the ICU. Using lumi-aggregometry, higher PR values in COVID-19 patients were found [6]. We used the MEA assay to assess PR in response to ADP, arachidonic acid, and TRAP. The MEA method measures the change in electrical impedance secondary to agonist-induced platelet aggregation in whole blood, which allows platelet function assessment under more physiological conditions than light transmission

Table 4 Coagulation results obtained with ROTEM ${ }^{\mathrm{TM}}$

\begin{tabular}{llllll}
\hline & $\boldsymbol{n}$ & COVID-19 & $\boldsymbol{n}$ & Control & $\boldsymbol{p}$ value \\
\hline CT (s), median (IQR) & 21 & $103.0(95.0-119.2)$ & 14 & $91.0(83.5-100.4)$ & 0.035 \\
CFT (s), median (IQR) & 21 & $64.0(56.2-73.0)$ & 14 & $78.0(67.5-89.0)$ & 0.047 \\
$\alpha$, median (IQR) & 21 & $77.0(75.0-81.0)$ & 14 & $74.5(72.7-77.2)$ & 0.027 \\
MCF, median (IQR) & 21 & $68.0(64.0-72.5)$ & 14 & $63.5(60.7-67.7)$ & 0.035 \\
ML (\%), median (IQR) & 21 & $14(10-45.5)$ & 14 & $36.5(13.5-56.7)$ & 0.092 \\
LI30 (\%), median (IQR) & 21 & $99.0(80.5-100.0)$ & 14 & $96.0(82.7-100.0)$ & 0.931 \\
\hline
\end{tabular}

$C T$ coagulation time, $C F T$ clot formation time, $\alpha$ alpha angle, $M C F$ maximum clot firmness, $M L$ maximum lysis, $L I 30$ lysis index $30 \mathrm{~min}$ after $\mathrm{CT}, I Q R$ interquartile range 
Table 5 Correlation between platelet reactivity and hospital length of stay $\leq 7$ of $>7$ days

\begin{tabular}{llll}
\hline Time hospitalization & MEA-ADP & MEA-ASPI & MEA-TRAP \\
\hline$\leq 7$ days $($ mean \pm SD) & $65.92 \pm 25.56$ & $68.76 \pm 26.18$ & $82.96 \pm 19.96$ \\
& $n=25$ & $n=25$ & $n=25$ \\
$>7$ days $($ mean \pm SD) & $45.91 \pm 19.0$ & $61.29 \pm 30.67$ & $63.58 \pm 17.74$ \\
& $n=34$ & $n=34$ & $n=31$ \\
$p$ value & 0.001 & 0.33 & $<0.001$ \\
\hline
\end{tabular}

$M E A$ multiplate electrode aggregometry, $A D P$ adenosine diphosphate, $A S P I$ aspirin, TRAP thrombin receptor-activating peptide 6, SD standard deviation

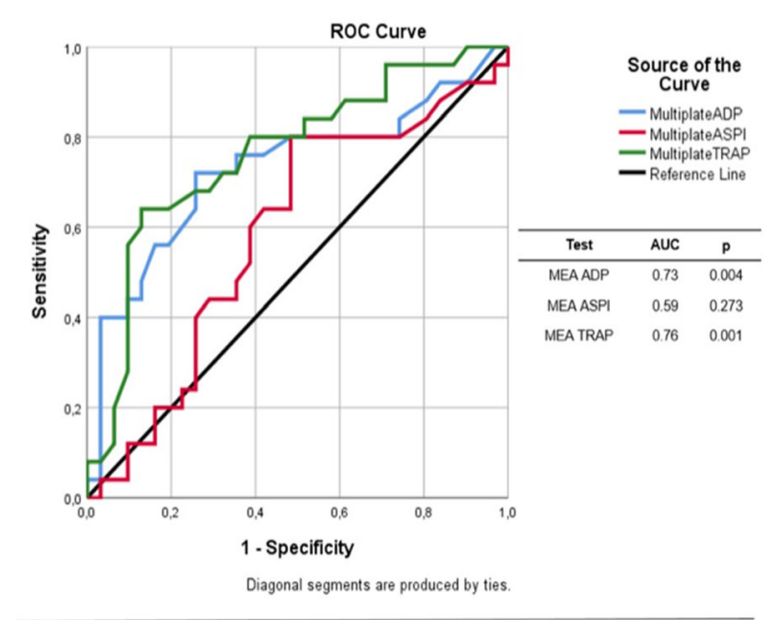

Receiver operating characteristic (ROC) curve for prediction of hospital length of stay more than 7 days based on platelet reactivity measured by multiplate electrode aggregometry (MEA) assay. ADP, adenosine diphosphate; ASPI, aspirin; TRAP, thrombin receptor-activating peptide.

Fig. 2 Correlation between platelet reactivity and hospital length of stay

aggregometry [20]. In addition, the MEA method has advantages over light transmission aggregometry, such as fewer laboratorial artifacts while handling blood samples [21]. The second study found that COVID-19 cases with moderate severity $(n=8)$ presented with exhausted platelets and a hyporeactive neutrophils phenotype, whereas severely affected patients $(n=9)$ had higher levels of platelet and neutrophil activation [7]. Moreover, analyzing 17 COVID-19 patients with Multiplate ADP, the authors found that $41 \%$ of them had platelet hyporeactivity (which is accordance with 33\% in our study).

Endothelial damage has been demonstrated in COVID-19, which can lead to a prothrombotic environment in the microcirculation and consequent activation of the coagulation cascade and platelet deposition [22]. Thus, our findings of platelet hypo-responsiveness observed in response to the arachidonic acid(cyxlooxygenase-thromboxane A pathway) and TRAP (thrombin pathways) by the MEA assay may be due to the fact that platelets during COVID-19 could be extensively activated in vivo, leading to subsequent refractoriness to new agonists added during ex vivo platelet function tests. This phenomenon (called "exhausted" platelets) has been described in patients with solid neoplasms, stroke, and sepsis, and has also been observed in patients with moderate to severe COVID-19 [23]. In addition, since most reactive platelets may be consumed in the microcirculation in COVID-19, platelets in the circulation may be less reactive. In this regard, decreased PR by MEA is also described in patients with sepsis [24]. Therefore, our results suggest that, in patients with COVID-19, continuous platelet activation in vivo may lead to a reduced response to activating agonists ex vivo.

Coagulation cascade disorders have been reported in COVID-19 patients [5, 25]. Similar to previous studies, our results demonstrated that patients with COVID-19 exhibited higher D-dimer and fibrinogen levels compared to healthy individuals [26, 27]. High levels of Ddimers were significantly associated with worse 
clinical outcome in COVID-19 [28]. Nonetheless, a recently published study did not observe a significant correlation between D-dimer and indicators of platelet reactivity among 115 consecutive COVID-19 patients presenting with respiratory symptoms [29].

As previously demonstrated, obesity is associated with increased PR and a lower antiplatelet effect of aspirin [30]. The fact that we had a higher number of obese patients in the COVID19 group reinforces our results, since the COVID-19 group was found to have lower PR compared to the control group.

We have found lower clot formation time, higher alfa angle, and an increased maximum clot firmness, indicating a hypercoagulable state. In addition, we have observed a trend for hypofibrinolysis-reduced lysis, suggestive of fibrinolysis shutdown in COVID-19, as previously demonstrated by different methodologies [26, 31-33].

Additionally, we have observed higher PAI-1 in COVID-19 patients. Previous analysis in SARS-CoV-1 revealed an upregulation of genes associated with the coagulation pathway, such as PAI-1 [34]. PAI-1 gene upregulation has been shown to inhibit fibrinolysis and to promote fibrin deposition during inflammatory states [35].

Thrombotic events are a main concern in patients with COVID-19, with the risk being higher in patients with more severe disease (up to $46 \%$ of deep vein thrombosis in 143 patients submitted to ultrasound scans of lower extremities in a routine basis), and leading to worse prognosis [36]. In our population, we had only 2 cases $(3.3 \%)$ of clinically diagnosed, thrombotic events which could be related to the lower risk of our population, and/or the fact that all patients were receiving prophylactic heparin.

Based on our observations, there is no basis for the use of antiplatelet drugs in the treatment of COVID-19. However, we will have a definite answer about such an important hypothesis (NCT04365309, NCT04363840, NCT04445623, NCT04409834) only with the results of ongoing studies.

Finally, to the best of our knowledge, ours is the first study to compare the length of hospitalization and PR in COVID-19 patients, showing that low PR has a good correlation and predictive value for longer hospitalization.

\section{Limitations}

In the present study, all COVID-19 patients were taking enoxaparin in prophylactic doses, which might have influenced PR. However, our group has previously demonstrated [37] in a population with stable coronary artery disease using low dose aspirin that the use of therapeutic dose enoxaparin ( $1 \mathrm{mg} / \mathrm{kg}$ BID) for 5 days did not significantly change PR, making this explanation unlikely.

Moreover, other drugs that may also influence PR were utilized in different proportions in the COVID-19 population; however, excluding each one of those drugs did not change our main results.

\section{CONCLUSION}

Patients hospitalized with non-severe COVID19 have lower PR compared to healthy controls, despite having higher levels of D-dimer, fibrinogen, and PAI-1, and hypercoagulable state by thromboelastometry. These data bring new important information in the understanding of the pathophysiology of COVID-19 and its complications.

\section{ACKNOWLEDGEMENTS}

We thank E. Colombo, D. Lourenco, C. Wroclawski and V. da Silva for technical assistance; J. Brandão, E. Moura and E. Marques for the assistance in collecting and storing the samples.

Funding. FAPESP (Fundação de Amparo à Pesquisa do estado de São Paulo) provided financial support (2020/04705-2). Siemens Healthcare Diagnostics, Newark, USA provided ADVIA Centaur Interleukin-6 immunoassay system. No Rapid Service Fee was received by the journal for the publication of this article. 
Authorship. All named authors meet the International Committee of Medical Journal Editors (ICMJE) criteria for authorship for this article, take responsibility for the integrity of the work as a whole, and have given their approval for this version to be published.

Author Contributions. J. Nicolau, A. Bertolin and T. Dalçoquio were responsible for the conception and design of the study; J. Nicolau, A. Bertolin, T. Dalçóquio, R. Salsoso and R.H.M. Furtado wrote the draft manuscript; A. Bertolin was responsible for the acquisition of data; $\mathrm{L}$. Baracioli, L. Hajjar, R. Kalil-Filho, R. Siciliano, E. Kallás, F. Lima, R. Giraldez, C. CavalheiroFilho, Robert P. Giugliano, U. Tantry, P. Gurbel contributed to the interpretation of the data and critically revised the draft manuscript; all authors gave final approval to the present manuscript. No editorial or any other assistance was utilized.

Disclosures. Remo H. de M. Furtado reports research grants and personal fees from AstraZeneca, Bayer; and Servier; and research grants from Pfizer, EMS, Aché, Brazilian Ministry of Health, and University Health Network. Roberto R. Giraldez reports personal fees, from Astra Zeneca, Boehringer Ingelheim, Daiichi Sankyo, Bayer, Pfizer and Merck. Udaya S.Tantry has received honoraria from UpToDate. Paul A. Gurbel has received consulting fees and/or honoraria from Bayer, Otitopic, Janssen, UpToDate, US WorldMeds, Hikari Dx, and Medicure; institutional research grants from the National Institutes of Health, Haemonetics, Bayer, Medicure, Instrumentation Laboratories, US WorldMeds, Amgen, Idorsia, Otitopic, and Janssen. Robert P. Giugliano reports grant support to his clinical trials from Amgen, Daiichi Sankyo, and Anthos Therapeutics, and honoraria for CME programs or consulting from Amgen, Daiichi Sankyo and Merck, consulting honoraria from Amarin, American College of Cardiology, Amgen, Astra Zeneca, BoehringerIngelheim, Bristol-Myers-Squibb, CryoLife, CVS Caremark, Dr. Reddy's Laboratories, Daiichi Sankyo, Eli Lilly and Company, Esperion, Gilead, GlaxoSmithKline, Janssen, Lexicon, Merck, Pfizer, St. Lukes, SAJA Pharmaceuticals,
Samsung and Servier. José C. Nicolau reports personal fees from AMGEN, grants from Astrazeneca, grants and personal fees from Bayer, grants from Esperion, grants from CLS Behring, personal fees from Daiichi-Sankyo, grants from Dalcor, grants from Janssen, grants and personal fees from Novartis, grants from NovoNordisk, grants and personal fees from Sanofi, personal fees from Servier, grants from Vifor, outside the submitted work; Adriadne J. Bertolin, Talia F. Dalçóquio, Rocío Salsoso, Luciano M. Baracioli, Felipe G. Lima, Roberto R. Giraldez, Cyrillo Cavalheiro-Filho, Alexandra Vieira, Célia M. C. Strunz, have nothing to disclose.

Compliance with Ethics Guidelines. This study was approved by the Institutional Review Board of the Ethics Committee (Comissão de Ética para Análise de Projetos de Pesquisa do HCFMUSP-CAAE: 34121120.2.0000.0068) and was conducted in accordance with the declaration of Helsinki of 1964 and its later amendments. All participants provided written informed consent. The study was registered at ClinicalTrials.gov (NCT04447131).

Data Availability. The datasets generated and/or analyzed during the current study are available from the corresponding author on reasonable request.

\section{REFERENCES}

1. Coronavirus resource center [Internet]. Available from: https://coronavirus.jhu.edu/.

2. Connors JM, Levy JH. COVID-19 and its implications for thrombosis and anticoagulation. Blood. 2020;135:2033-40.

3. Choudry FA, Hamshere SM, Rathod KS, Akhtar MM, Archbold RA, Guttmann OP, et al. High thrombus burden in patients with COVID-19 presenting with ST-segment elevation myocardial infarction. J Am Coll Cardiol. 2020;76:1168-76.

4. Ren B, Yan F, Deng Z, Zhang S, Xiao L, Wu M, et al. Extremely high incidence of lower extremity deep venous thrombosis in 48 patients with severe COVID-19 in Wuhan. Circulation. 2020;142:181-3. 
5. Levi M, Thachil J, Iba T, Levy JH. Coagulation abnormalities and thrombosis in patients with COVID-19. Lancet Haematol. 2020;7:e438-40.

6. Manne BK, Denorme F, Middleton EA, Portier I, Rowley JW, Stubben C, et al. Platelet gene expression and function in patients with COVID-19. Blood. 2020;10(136):1317-29.

7. Nicolai L, Leunig A, Brambs S, Kaiser R, Weinberger $\mathrm{T}$, Weigand $\mathrm{M}$, et al. Immunothrombotic dysregulation in COVID-19 pneumonia is associated with respiratory failure and coagulopathy. Circulation. 2020;142:1176-89.

8. Viecca M, Radovanovic D, Forleo GB, Santus P. Enhanced platelet inhibition treatment improves hypoxemia in patients with severe Covid-19 and hypercoagulability. A case control, proof of concept study. Pharmacol Res. 2020;158:104950.

9. Liu Y, Sun W, Guo Y, Chen L, Zhang L, Zhao S, et al. Association between platelet parameters and mortality in coronavirus disease 2019: retrospective cohort study. Platelets. 2020;7104:1-7.

10. Yang X, Yang Q, Wang Y, Wu Y, Xu J, Yu Y, et al. Thrombocytopenia and its association with mortality in patients with COVID-19. J Thromb Haemost. 2020;18:1469-72. https://doi.org/10.1111/ jth. 14848 .

11. Würtz M, Hvas AM, Christensen KH, Rubak $P$, Kristensen SD, Grove EL. Rapid evaluation of platelet function using the Multiplate ${ }^{\circledR}$ analyzer. Platelets. 2014;25:628-33.

12. Crochemore $\mathrm{T}$, de $\mathrm{T}$ Piza FM, Rodrigues RDR, de $\mathrm{C}$ Guerra JC, Ferraz LJR, Corrêa TD. A new era of thromboelastometry. Einstein (Sao Paulo). 2017;15: $380-5$.

13. Peng HT, Nascimento B, Beckett A. Thromboelastography and thromboelastometry in assessment of fibrinogen deficiency and prediction for transfusion requirement: a descriptive review. Biomed Res Int. 2018;2018:7020539.

14. Thygesen K, Alpert JS, Jaffe AS, Chaitman BR, Bax JJ, Morrow DA, et al. Fourth universal definition of myocardial infarction (2018). Eur Heart J. 2019;40: 237-69.

15. Konstantinides SV, Meyer G, Bueno H, Galié N, Gibbs JSR, Ageno W, et al. 2019 ESC Guidelines for the diagnosis and management of acute pulmonary embolism developed in collaboration with the European Respiratory Society (ERS). Eur Heart J. 2020;41:543-603.

16. Rubak P, Villadsen K, Hvas A-M. Reference intervals for platelet aggregation assessed by multiple electrode platelet aggregometry. Thromb Res. 2012;1(130):420-3.

17. Cells NT. ASPItest 08847533190. 2019;4-7.

18. Cells NT. TRAPtest 08847509190. 2019;6-9.

19. Cells NT. ADPtest 08847550190. 2019.

20. Paniccia R, Priora R, Liotta AA, Abbate R. Platelet function tests: a comparative review. Vasc Health Risk Manag. 2015;11:133-48.

21. McGlasson DL, Fritsma GA. Whole blood platelet aggregometry and platelet function testing. Semin Thromb Hemost. 2009;35:168-80.

22. Roberts KA, Colley L, Agbaedeng TA, EllisonHughes GM, Ross MD. Vascular manifestations of COVID-19-thromboembolism and microvascular dysfunction. Front Cardiovasc Med. 2020;7:598400.

23. van der Meijden PEJ, Heemskerk JWM. Platelet biology and functions: new concepts and clinical perspectives. Nat Rev Cardiol. 2019;16:166-79.

24. Adamzik M, Görlinger K, Peters J, Hartmann M. Whole blood impedance aggregometry as a biomarker for the diagnosis and prognosis of severe sepsis. Crit Care. 2012;16:R204.

25. Al-Samkari H, Karp Leaf RS, Dzik WH, Carlson JCT, Fogerty AE, Waheed A, et al. COVID-19 and coagulation: bleeding and thrombotic manifestations of SARS-CoV-2 infection. Blood. 2020;136:489-500.

26. Spiezia L, Boscolo A, Poletto F, Cerruti L, Tiberio I, Campello E, et al. COVID-19-related severe hypercoagulability in patients admitted to intensive care unit for acute respiratory failure. Thromb Haemost. 2020;120:998-1000.

27. Helms J, Tacquard C, Severac F, Leonard-Lorant I, Ohana M, Delabranche $X$, et al. High risk of thrombosis in patients with severe SARS-CoV-2 infection: a multicenter prospective cohort study. Intensive Care Med. 2020;46:1089-98.

28. Tang N, Li D, Wang X, Sun Z. Abnormal coagulation parameters are associated with poor prognosis in patients with novel coronavirus pneumonia. J Thromb Haemost. 2020;18:844-7.

29. Younes Z, Florian P, Isabelle A, Abdallah N, Mounia $\mathrm{O}$, Loubna $\mathrm{K}$, et al. Platelets can associate with SARS-Cov-2 RNA and are hyperactivated in COVID19. Circ Res. 2020;127:1404-18.

30. Mourikis P, Zako S, Dannenberg L, Helten C, Naguib D, Hohlfeld T, et al. Aspirin antiplatelet effects are associated with body weight. Vascul Pharmacol. 2020;125-126:106635. 
31. Wright FL, Vogler TO, Moore EE, Moore HB, Wohlauer MV, Urban S, et al. Fibrinolysis shutdown correlation with thromboembolic events in severe COVID-19 infection. J Am Coll Surg. 2020;231:193203.e1.

32. Pavoni V, Gianesello L, Pazzi M, Stera C, Meconi T, Frigieri FC. Evaluation of coagulation function by rotation thromboelastometry in critically ill patients with severe COVID-19 pneumonia. J Thromb Thrombolysis. 2020;50:281-6.

33. Yuriditsky E, Horowitz JM, Merchan C, Ahuja T, Brosnahan SB, McVoy L, et al. Thromboelastography profiles of critically ill patients with coronavirus disease. Crit Care Med. 2019;2020:1319-26.

34. Tang BSF, Chan KH, Cheng VCC, Yuen KY. Comparative host gene transcription by microarray analysis early after infection of the Huh7 cell line by SARS coronavirus and human coronavirus $229 \mathrm{E}$. Hong Kong Med J. 2009;15(SUPP9):23-6.

35. Katayama S, Koyama K, Shima J, Tonai K, Goto Y, Koinuma $\mathrm{T}$, et al. Thrombomodulin, plasminogen activator inhibitor-1 and protein C levels, and organ dysfunction in sepsis. Crit Care Explor. 2019;1:e0013.

36. Zhang L, Feng X, Zhang D, Jiang C, Mei H, Wang J, Zhang C, Li H, Xia X, Kong S, et al. Deep vein thrombosis in hospitalized patients with COVID-19 in Wuhan, China. Circulation. 2020;142:114-28.

37. Arantes FB, Menezes FR, Franci A, Barbosa CJ, Dalçoquio TF, Nakashima CA, Baracioli LM, Furtado $\mathrm{RH}$, Nomelini QS, Ramires JA, et al. Influence of direct thrombin inhibitor and low molecular weight heparin on platelet function in patients with coronary artery disease: a prospective interventional trial. Adv Therapy. 2019;37:420-30. 